\title{
Probing Long Range Scalar Dark Forces in Terrestrial Experiments
}

\author{
Sonny Mantry ${ }^{1}$ \\ University of Wisconsin at Madison,WI,53705,USA
}

\begin{abstract}
A long range Weak Equivalence Principle (WEP) violating force between Dark Matter (DM) particles, mediated by an ultralight scalar, is tightly constrained by galactic dynamics and large scale structure formation. We examine the implications of such a "dark force" for several terrestrial experiments, including Eötvös tests of the WEP and direct-detection DM searches. The presence of a dark force implies a non-vanishing effect in Eötvös tests that could be probed by current and future experiments depending on the DM model. For scalar singlet DM scenarios, a dark force of astrophysically relevant magnitude is ruled out in large regions of parameter space by the DM relic density and WEP constraints. WEP tests also imply constraints on the Higgs-exchange contributions to the spin-independent (SI) DM-nucleus direct detection cross-section. For WIMP scenarios, these considerations constrain Higgs-exchange contributions to the SI cross-section to be subleading compared to gauge-boson mediated contributions. The combination of observations from galactic dynamics, large scale structure formation, Eötvös experiments, DM-direct-detection experiments, and colliders can further constrain the size of new long range forces in the dark sector.
\end{abstract}

Keywords: Dark forces, Dark Matter, Weak Equivalence Principle, Eotvos

PACS: $<95.35 .+\mathrm{d}, 98.80 . \mathrm{Cq}>$

\section{INTRODUCTION}

Despite the large body of evidence for Dark Matter (DM) from the galactic rotation curves [1, 2], acoustic oscillations in the cosmic microwave background [3, 4, 5, 6], large scale structure formation [7, 8], and gravitational lensing [9, 10] almost nothing is known about the properties of DM and a great deal of experimental effort is under way to unravel its nature. Ground based direct detection experiments [11,12] put limits on the DM mass and the strength of its interaction with baryonic matter from observations of recoiling nuclei. Experiments [13, 14, 15, 16] studying cosmic rays from the galactic halo have recently seen indications of an electron/positron excess, which could be interpreted as evidence for DM annihilation, and can constrain the DM mass and interactions.

Another set of experiments are devoted to question of whether DM violates the Weak Equivalence Principle (WEP). Such an apparent violation of the WEP can occur in the presence of a new long range scalar force coupled to DM and is the focus of this work. In particular, we consider the possibility of an ultralight scalar with mass $m_{\phi}<10^{-25}$ $\mathrm{eV}$ coupling directly to DM with intergalactic range. There are a few hints for the presence of a dark force from the higher than predicted supercluster densities [17], and voids [18, 19], and galaxy clusters [20] ( for a summary see [21, 22]). Strong constraints on a dark force are derived from observations of DM dynamics in the tidal stream of the Sagittarius dwarf galaxy [21, 23], which indicate a force with strength less that $20 \%$ of gravity. However, new observational systematic errors have been recently discovered [24] that could require a revision of this result, perhaps allowing for a stronger dark force. A more recent analysis [25] considers the effect of a dark force on the evolution of density perturbations and the resulting impact on the CMB spectrum with similar constraints.

Many models that contain the interaction of an ultralight scalar with DM [26, 27, 28, 29, 30, 31, 18, 32, 33, 34, 19, 35] have been proposed to explain features in the DM distribution and explore the possibility of DM-quintessence interactions. More recently, work with non-universal scalar-tensor theories of gravity with the Abnormally Weighting Energy (AWE) Hypothesis [36, 37] also invoke couplings of an ultralight scalar to the dark sector as a way of explaining the observed cosmic acceleration even in the absence of a dark energy fluid. Constraints on such scenarios from big bang nucleosynthesis have also been studied [38].

If the DM has interactions with the Standard Model (SM), the presence of a scalar dark force will be communicated

\footnotetext{
${ }^{1}$ Contributed talk at the Invisible Universe Conference, 2009, Paris, France.
} 

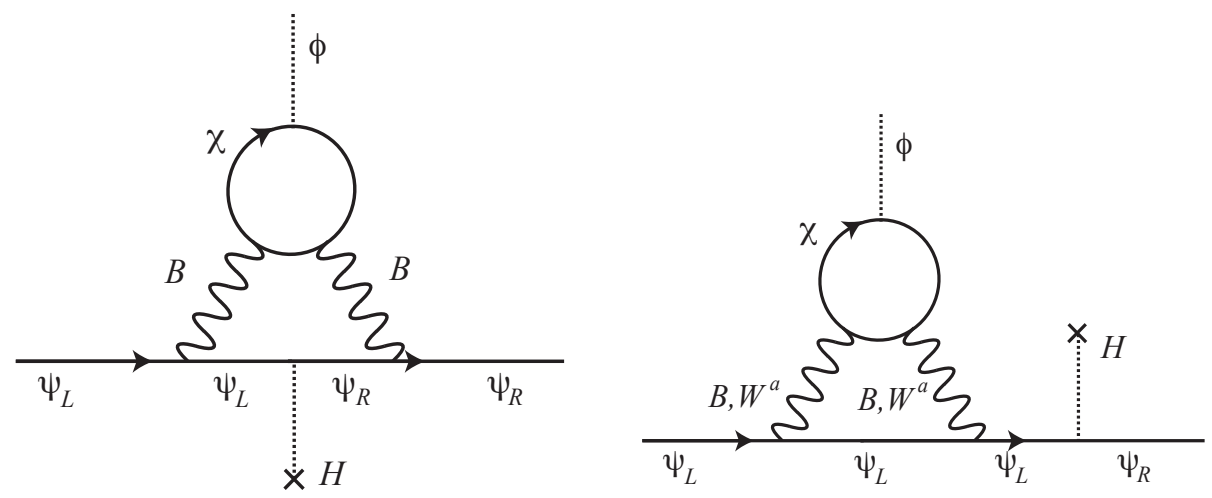

FIGURE 1. Two-loop diagrams in WIMP DM models that generate the operators $\mathscr{O}_{f}^{H}$ in Eq. 7 which generate couplings of the ultralight scalar to the SM fermions after electroweak symmetry breaking.

to the SM via virtual DM. This effect can be exploited to connect signatures of dark forces in astrophysics and cosmology with terrestrial experiments such as the laboratory Eötvös tests of the WEP, DM-direct-detection experiments, and in some cases even at colliders. This connection has been studied in [22] and [39] and more recently an extensive analysis was done in [40] and is the focus of this note.

\section{WEP VIOLATION PHENOMENOLOGY}

We first discuss some basic phenomenology of new long range Yukawa forces coupling to ordinary matter or DM. The potential $V$ between two bodies of mass $M_{i}$ and $M_{s}$, in the presence of a new long range Yukawa force mediated by a scalar of mass $m_{\phi}$, can be parameterized as

$$
V=-\frac{G M_{i} M_{s}}{r}\left(1+\alpha_{i s} e^{-m_{\phi} r}\right), \quad \alpha_{i s}=\frac{1}{4 \pi G} \frac{Q_{i} Q_{s}}{M_{i} M_{s}} \xi_{i} \xi_{s},
$$

where the first term in the potential is the usual Newtonian gravitational potential and the the parameter $\alpha_{i s}$ is a product of the charge to mass ratios $Q_{i, s} / M_{i, s}$ of the two bodies under the new Yukawa force. The factors of $\xi_{i, s}$ appear for dimensional reasons [40] and are 1 for fermionic test objects and $1 /\left(2 M_{i, s}\right)$ for scalar test objects. The Eötvös parameter $\eta_{s}$ which measures the relative acceleration between two test objects in the presence of a gravitational source $s$ is given by

$$
\eta_{s}=2 \frac{\left|a_{1}-a_{2}\right|}{\left|a_{1}+a_{2}\right|} \simeq \frac{1}{4 \pi G}\left|\frac{Q_{1} \xi_{1}}{M_{1}}-\frac{Q_{2} \xi_{2}}{M_{2}}\right|\left|\frac{Q_{s} \xi_{s}}{M_{s}}\right|,
$$

where we ignored the small mass $m_{\phi}<10^{-25} \mathrm{eV}$ for the ranges $r \ll m_{\phi}^{-1}$ being probed. The current bounds [41] on the Eötvös parameters $\eta_{E}^{\mathrm{Be}, \mathrm{Ti}}$ and $\eta_{D M}^{\mathrm{Be}, \mathrm{Ti}}$ which measure the relative acceleration of macroscopic samples of Beryllium and Titanium toward the center of the Earth and galactic DM respectively are

$$
\eta_{E}^{B e, T i}<(0.3 \pm 1.8) \times 10^{-13} \quad \eta_{D M}^{B e, T i}<(4 \pm 7) \times 10^{-5} .
$$

The Apollo (LLR) [42] and Microscope[43] collaborations are expected to be sensitive to values of $\eta_{E}^{\mathrm{Be}, \mathrm{Ti}}$ as small as $10^{-14}$ and $10^{-15}$ respectively. The MiniSTEP[44] proposal, if approved, can be sensitive to values of $\eta_{E}^{\mathrm{Be}, \mathrm{Ti}}$ as small as $10^{-18}$.

We parameterize the coupling of the ultralight scalar to DM by

$$
\delta \mathscr{L}= \begin{cases}g_{\chi} \bar{\chi} \chi \phi, & \text { fermionic DM, } \\ g_{\chi} \chi^{\dagger} \chi \phi, & \text { scalar DM, }\end{cases}
$$



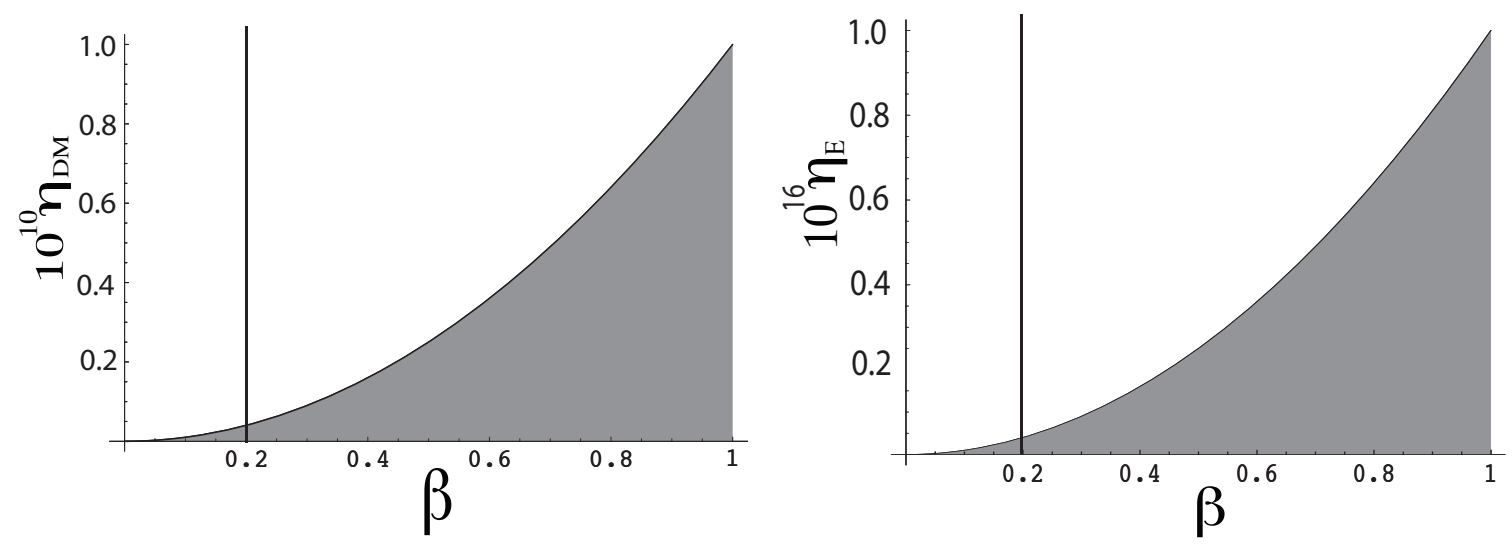

FIGURE 2. An estimate of the allowed region in the $\left(\eta_{D M, E}, \beta\right)$ parameter space for minimal WIMP DM models. The curves in the figures give an estimate of for $\eta_{D M . E}$ for a given value of $\beta$ from the two loop diagrams in Fig. 1 The shaded region is unlikely for typical WIMP models. Using the observational constraint $\beta<0.2$, the allowed region is further restricted to the left of the vertical line. The estimates in the above figures for $\eta_{D M . E}^{\mathrm{Be}, \mathrm{Ti}}$, for $\beta<0.2$, are far below the current experimental bounds $\eta_{D M}^{\mathrm{Be}, \mathrm{Ti}}<10^{-5}, \eta_{E}<10^{-13}$. An improvement of about five orders of magnitude would be required in Eötvös experiments to fully probe the allowed parameter space for $\beta=0.2$ by measuring $\eta_{E}^{\mathrm{Be}, \mathrm{Ti}}$. This is within reach of the MiniSTEP proposal[44] .

where $\chi$ denotes the DM field and $g_{\chi}$ is the coupling of DM to the ultralight scalar. For simplicity we assume that fermionic DM lives in vector-like representations of the electroweak gauge group so that the interaction is gauge invariant. For chiral DM the interaction with the ultralight scalar can arise only from higher dimension operators. From observations in astrophysics [21, 23] and cosmology [25], the most recent bound on the charge to mass ratio of DM under the new dark force parameterized by $\beta$ is given as

$$
\beta=\frac{M_{P}}{\sqrt{4 \pi}} \frac{\left|g_{\chi}\right|}{M_{\chi}} \xi_{\chi}, \quad \beta<0.2,
$$

where $M_{P}$ denotes the Planck Mass. This bound corresponds to a dark force restricted to be less than about $20 \%$ of gravity.

\section{SCALAR DARK FORCES AND TERRESTRIAL EXPERIMENTS}

If the DM has interactions with the SM then the bounds on the parameters $\eta_{E, D M}^{\mathrm{Be}, \mathrm{Ti}}$ and $\beta$, obtained from laboratory WEP tests and astrophysical and cosmological observations respectively, will in general be correlated. As a simple example consider minimal WIMP DM models [45] of the type

$$
\mathscr{L}= \begin{cases}\bar{\chi}\left(i D+M_{0}\right) \chi, & \text { fermionic DM, } \\ c\left(D_{\mu} \chi\right)^{\dagger} D^{\mu} \chi-c M_{0}^{2} \chi^{\dagger} \chi-V(\chi, H), & \text { scalar DM, }\end{cases}
$$

where a single DM particle $\chi$ is added to the SM with non-zero electroweak interactions. Relic density considerations typically require the $\mathrm{DM}$ mass to be in the $\mathrm{TeV}$ range in such models. We point out that for non-zero hypercharge, such minimal WIMP DM models are typically ruled out [45] by direct detection experiments. Here we consider such minimal DM models, even with non-zero hypercharge, only as illustrative examples keeping in mind that such DM could be part of a non-minimal extension which avoids the direct detection bounds. If the DM couples directly to a dark force as in Eq. (4), then this dark force will couple to the SM quarks and leptons via two diagrams as shown in Fig. 1. In particular, before electroweak symmetry breaking these loop diagrams generate the dimension five operators of the type

$$
\mathscr{O}_{u}^{H}=\phi \bar{Q}_{L} \varepsilon H^{\dagger} C_{u}^{H} u_{R}, \quad \mathscr{O}_{d}^{H}=\phi \bar{Q}_{L} H C_{d}^{H} d_{R}, \quad \mathscr{O}_{e}^{H}=\phi \bar{L}_{L} H C_{e}^{H} e_{R},
$$




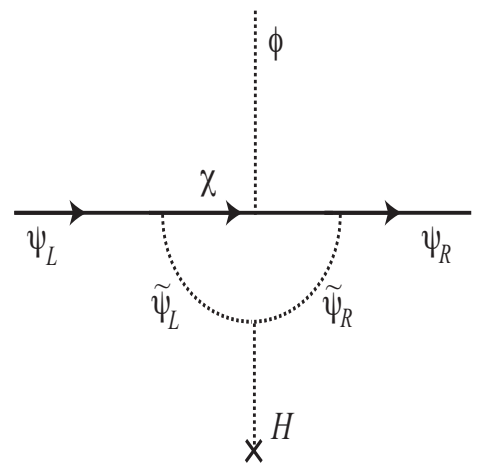

FIGURE 3. DM-induced coupling of $\phi$ to SM fermions at one loop in the presence of additional squark and slepton like degrees of freedom.

where the coefficients $C_{u, d, e}^{H}$ are finite and computable from the loop diagrams. After electroweak symmetry breaking, the Higgs field gets a vacuum expectation value so that these operators generate a coupling between the ultralight scalar and the SM fermions. Note that the coupling of the ultralight scalar to the SM fermions must come from induced higher dimension operators since no gauge invariant renormalizable couplings exist between SM fermions and a gauge singlet scalar before electroweak symmetry breaking. From the induced coupling of the ultralight scalar to the SM model fermions one can obtain order of magnitude estimates [40] for the Eötvös parameters in terms of the parameter $\beta$. For minimal WIMP models of the type in Eq. $\sqrt{6}$, the lower bound on the Eötvös parameters $\eta_{E, D M}^{\mathrm{Be}, \mathrm{Ti}}$ as a function of $\beta$ is plotted in Fig. 2. For $\beta=0.2$, marked by the vertical lines in Fig. 2, we see that the lower bounds for typical WIMP DM models are $\eta_{D M}^{\mathrm{Be}, \mathrm{Ti}}>4 \times 10^{-12}$ and $\eta_{E}^{\mathrm{Be}, \mathrm{Ti}}>4 \times 10^{-18}$. These lower bounds are far below the current experimental upper bounds shown in Eq. (3). An improvement of about five to seven orders of magnitude in Eötvös experiments would be required in order to probe these lower bounds for WIMP DM models. The MiniSTEP [44] experiment, which is currently under study, is expected to reach a sensitivity for $\eta_{E}^{\mathrm{Be}, \mathrm{Ti}}$ of about $10^{-18}$ and might be able to probe the lower bounds. If an effect is detected in $\eta_{D M, E}^{\mathrm{Be}, \mathrm{Ti}}$ far above the lower bounds in Fig. 2 . it would suggest the possibility that the coupling of $\phi$ to the SM fermions might have a different origin. Another possibility that might explain an effect above the expectation in Fig. 2 would be a stronger induced coupling of $\phi$ to ordinary matter arising from non-minimal DM models; for example a one loop coupling of $\phi$ to ordinary matter (see Fig. 3 in the presence of additional squark degrees of freedom [40]. The main point is that given a DM model one can estimate the expected size for the Eötvös parameters $\eta_{E, D M}$ for a given value of $\beta$ thus correlating observations in astrophysics and cosmology with laboratory WEP tests.

Another mechanism by which the ultralight scalar can couple to SM fermions is by mixing with the Higgs. While the ultralight scalar cannot have any renormalizable couplings to the SM fermions before electroweak symmetry breaking, it can have direct couplings to the SM Higgs. After electroweak symmetry breaking, the ultralight scalar will mix with the SM Higgs and in turn couple to the SM fermions. The size of this mixing angle will be tightly constrained by Eötvös tests since such an induced coupling to the SM fermions will lead to a violation of the WEP. In what follows we will refer to the ultralight scalar gauge eigenstate as $S$ and the mass eigenstate after electroweak symmetry breaking as $\phi$. The renormalizable couplings of the ultralight scalar $S$ to the SM Higgs doublet are encoded in the potential

$$
\begin{aligned}
V(H, S) & =-\mu_{h}^{2} H^{\dagger} H+\frac{\lambda}{4}\left(H^{\dagger} H\right)^{2}+\frac{\delta_{1}}{2} H^{\dagger} H S+\frac{\delta_{2}}{2} H^{\dagger} H S^{2} \\
& -\left(\frac{\delta_{1} \mu_{h}^{2}}{\lambda}\right) S+\frac{\kappa_{2}}{2} S^{2}+\frac{\kappa_{3}}{3} S^{3}+\frac{\kappa_{4}}{4} S^{4} .
\end{aligned}
$$

We assume for simplicity that $S$ does not acquire a vaccum expectation value. After electroweak symmetry breaking the $H^{\dagger} H S$ interaction induces mixing between the Higgs boson and the ultralight scalar. In unitary gauge the neutral component of the Higgs doublet $H$ is given by

$$
H^{0}=\frac{v+h}{\sqrt{2}}, \quad v=\sqrt{\frac{2 \mu_{h}^{2}}{\lambda}},
$$




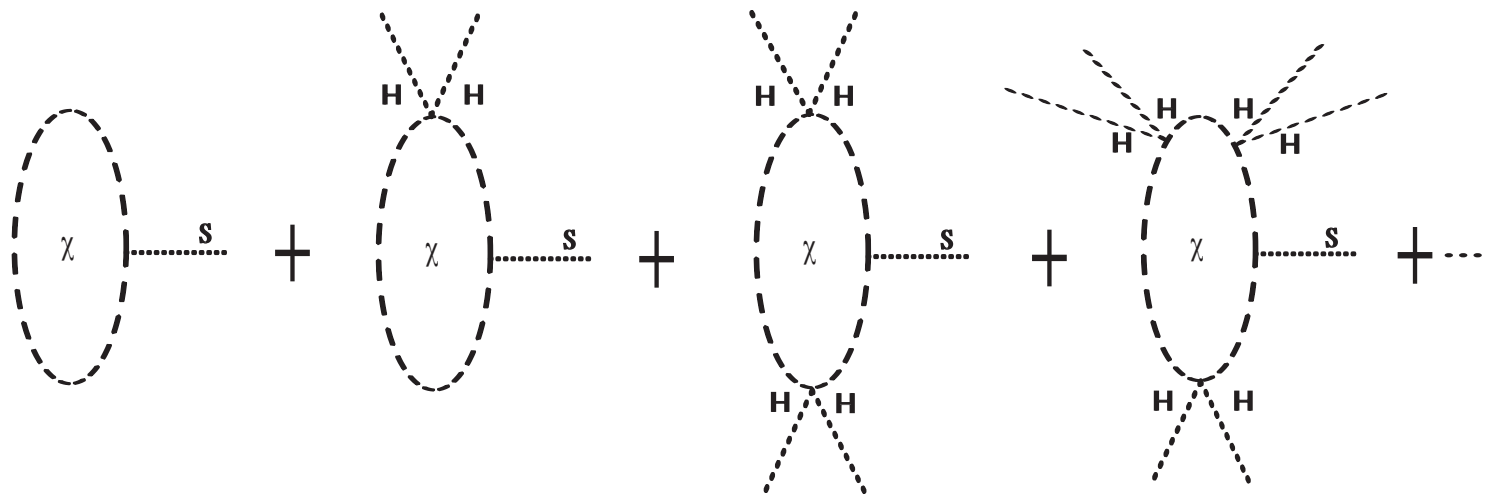

FIGURE 4. One loop diagrams which which contribute to terms in the effective potential $V(H, S)$ that are linear in the $S$ field. The first two diagrams are UV divergent and contribute to the renormalization of the $S$-tadpole and the coupling $\delta_{1}$ respectively. The remaining diagrams mix into higher dimensional operators and give a finite contribution to Higgs-ultralight-scalar mixing after electroweak symmetry breaking.

and the mass terms in the potential become

$$
V_{\text {mass }}=\frac{1}{2}\left(\mu_{h}^{2} h^{2}+\mu_{S}^{2} S^{2}+\mu_{h S}^{2} h S\right)
$$

where

$$
\mu_{h}^{2}=\frac{\lambda v^{2}}{2}, \quad \mu_{S}^{2}=\kappa_{2}+\frac{\delta_{2} v^{2}}{2}, \quad \mu_{h S}^{2}=\delta_{1} v .
$$

The mass eigenstates $h_{ \pm}$in terms of $S$ and $h$ can be written in terms of a mixing angle $\theta$ as

$$
h_{-}=S \cos \theta-h \sin \theta, \quad h_{+}=S \sin \theta+h \cos \theta, \quad \tan \theta=\frac{x}{1+\sqrt{1+x^{2}}},
$$

with corresponding masses

$$
m_{ \pm}^{2}=\frac{\mu_{h}^{2}+\mu_{S}^{2}}{2} \pm \frac{\mu_{h}^{2}-\mu_{S}^{2}}{2} \sqrt{1+x^{2}}, \quad x \equiv \frac{\mu_{h S}^{2}}{\mu_{h}^{2}-\mu_{S}^{2}} .
$$

We identify $h_{+}$and $m_{+}$with the physical Higgs boson field and mass respectively and the physical ultralight scalar field and mass are identified as

$$
\phi \equiv h_{-}, \quad m_{\phi}=m_{-} .
$$

Now if the DM couples to the ultralight scalar as in Eq. (4), the mixing angle $\theta$ could receive additional contributions from virtual DM effects. As a concrete example consider the DM model where the DM $\chi$ is either a real singlet or real triplet scalar under the electroweak gauge group with potential

$$
V(H, S, \chi)=V(H, S)+\frac{1}{2} M_{0}^{2} \chi^{2}+\frac{\lambda_{\chi}}{4} \chi^{4}+a_{2} H^{\dagger} H \chi^{2}+g_{\chi} \chi^{2} S+\lambda_{\chi s} \chi^{2} S^{2},
$$

where we have imposed the $Z_{2}$ symmetry $\chi \rightarrow-\chi$ for the DM field to ensure its stability. The experimental constraints on this DM model in the absence of a dark force were studied in [46, 47]. The parameter $a_{2}$ determines the spinindependent cross-section for DM direct detection

$$
\sigma_{\chi N} \simeq \frac{a_{2}^{2} g_{h}^{2} v^{2} m_{N}^{2}}{\pi\left(M_{\chi}+m_{N}\right)^{2} m_{h}^{4}}, \quad M_{\chi}^{2}=M_{0}^{2}+a_{2} v^{2}
$$



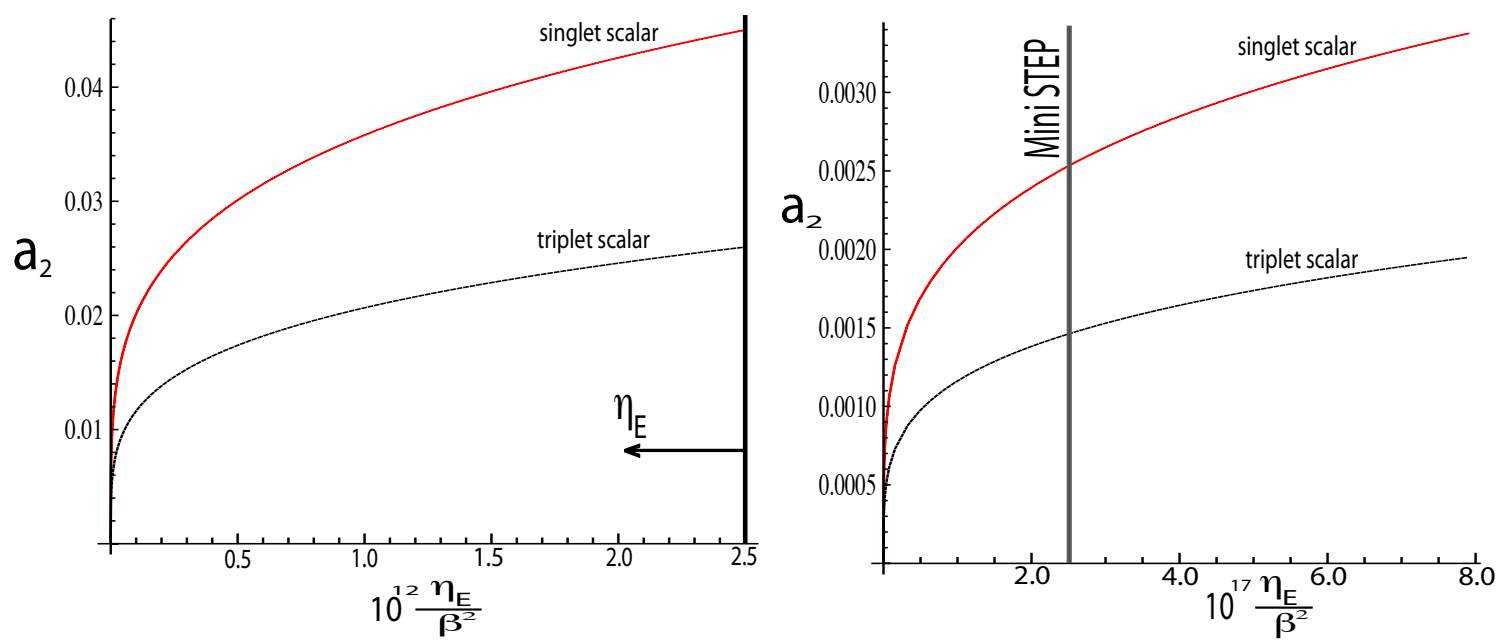

FIGURE 5. Upper bound on $a_{2}$ in the singlet (upper curve) and real triplet (lower curve) scalar DM models as a function of $\eta_{E}^{\mathrm{Be}, \mathrm{Ti}} / \beta^{2}$. The vertical black line in the left panel corresponds to the current bound of $\eta_{E}^{B e, T i}<(0.3 \pm 1.8) \times 10^{-13}$. This vertical black line will move to the left with further improvements in Eötvös experiments as indicated by the arrow pointing to the left. The right panel shows the region closer to the expected future bounds from the MiniSTEP experiment and the vertical black line here corresponds to the expected sensitivity of MINISTEP of $\eta_{E}^{B e, T i}=10^{-18}$. We have used $m_{h}=120 \mathrm{GeV}$ and $\beta=0.2$.

where $M_{\chi}$ is the physical DM mass after electroweak symmetry breaking. For the singlet DM model, $a_{2}$ also determines the relic density as a function of the Higgs mass. We now show that the presence of a dark force can imply an upper bound on the magnitude of $a_{2}$ which can translate into an upper bound on the spin-independent direct detection crosssection. For the singlet DM model, the implied upper bound on the magnitude of $a_{2}$ can be used to rule out dark forces in large regions of parameter space from the DM relic density constraint.

It was shown in [40] that the mixing angle between the ultralight scalar and the Higgs is of the form

$$
\sin \theta \approx \tan \theta \approx x \approx \kappa \frac{a_{2}^{2}}{\pi^{3 / 2}} \frac{v^{3}}{M_{P} m_{h}^{2}} \beta+\frac{\delta_{1} v}{m_{h}^{2}}
$$

where $\kappa=1,3$ for the real singlet and real triplet DM respectively. The second term on the RHS above comes from the renormalizable term $H^{\dagger} H S$ term after electroweak symmetry breaking. The first term on the RHS in Eq.(17) is the result of summing the contributions of higher dimension operators of the form $\left(H^{\dagger} H\right)^{n} S$, for $n \geq 2$, induced via virtual DM as shown in Fig 4 at one loop. The first two diagrams in Fig. 4 are UV divergent and just contribute to renormalization of the $S$-tadpole and the $\delta_{1}$ coupling respectively. The remaining diagrams are finite and induce operators of the form $\left(H^{\dagger} H\right)^{n} S$ which give rise to the first term on the RHS of Eq. 117) after electroweak symmetry breaking. For more details see [40].

As mentioned earlier, Eötvös experiments will constrain the size of $\sin \theta$ since it leads to WEP violation for ordinary matter and will generate a non-zero $\eta_{E, D M}^{\mathrm{Be}, \mathrm{Ti}}$. Furthermore, note that the RHS of Eq. $\sqrt{17}$ involves the parameter $\beta$ which is constrained from cosmology and astrophysics as in Eq. (5). Except in slices of parameter space where there are intricate cancellations between the two terms on the RHS of Eq.(17), which is unlikely given that $\delta_{1}$ and $a_{2}$ are not related in any way, one can use the Eötvös tests to put an upper bound [40] on the magnitude of $a_{2}$ as shown in Fig. 5 Figure 5 shows the upper bound on the magnitude of $a_{2}$ as a function of the ratio $\eta_{E}^{\mathrm{Be}, \mathrm{Ti}} / \beta^{2}$. In both panels of Fig. 5 the upper curve is for the singlet scalar DM model and the lower curve is for the real triplet scalar DM model. The vertical black line on the right end of the left panel in Fig. 5 corresponds to the values $\eta_{E}^{B e, T i}=(0.3 \pm 1.8) \times 10^{-13}$ and $\beta=0.2$ which are the current bounds. The vertical black line in the right panel in Fig. 5 corresponds to the value $\eta_{E}^{B e, T i}=10^{-18}$, which is the expected sensitivity for the MINISTEP proposal, and $\beta=0.2$. For singlet scalar DM, the upper bound on $a_{2}$ from Fig. 5 can be compared with the required value of $a_{2}$ [47] for a given Higgs mass in order to get the right DM relic density and thus further constrain dark forces. For example, for a Higgs mass of $120 \mathrm{GeV}$ and singlet scalar DM mass of $20 \mathrm{GeV}$, the required value for $a_{2}$ is about 0.15 . In this case a dark force with strength corresponding $\beta=0.2$ is ruled out by the current bound on $\eta_{E}^{\mathrm{Be}, \mathrm{Ti}}$ shown in Eq. 3 . This can be since in the left panel of Fig. 5. which requires $a_{2}<0.045$ as seen from the intersection of the upper curve with the vertical line on the right. 

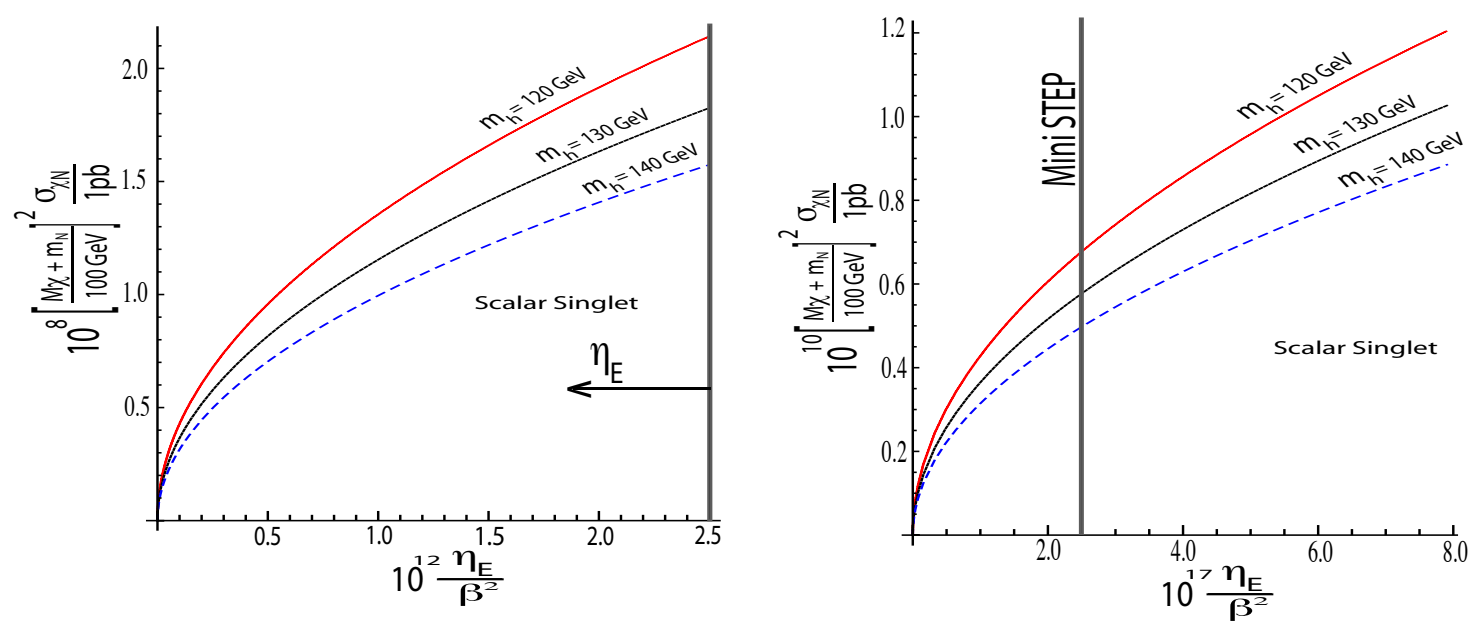

FIGURE 6. Upper bounds on the spin-independent direct detection cross-section for the scalar singlet DM model as a function of $\eta_{E}^{\mathrm{Be}, \mathrm{Ti}} / \beta^{2}$. The vertical line in the left panel corresponds to the current bound of $\eta_{E}^{B e, T i}<(0.3 \pm 1.8) \times 10^{-13}$. This vertical line will move to the left with further improvements in Eötvös experiments as indicated by the arrow pointing to the left. The right panel shows the region closer to the expected future bounds from the MiniSTEP experiment and the vertical line here corresponds to the expected sensitivity of MINISTEP of $\eta_{E}^{B e, T i}=10^{-18}$. We have used $\beta=0.2$ and the tree curves in each panel correspond to Higgs mass values of $m_{h}=120,130,140 \mathrm{GeV}$ as indicated.

From Eq.[16, the upper bound on the magnitude of $a_{2}$ also translates into an upper bound on the spin-independent cross-section as shown in Fig. 6for the singlet DM model. For brevity we have not shown the corresponding plots [40] for the real triplet DM model. Figure 6 shows how the spin-independent DM direct detection cross-section, Eötvös tests, and observations in astrophysics and cosmology can be brought together to constrain dark forces. As seen in Fig. 6. smaller values of $\beta$ for a given value of $\eta_{E}^{\mathrm{Be}, \mathrm{Ti}}$, lead to a weaker bound allowing for larger values of the spinindependent direct detection cross-section. As seen from the left panel in Fig. 6 , for a DM mass of around $100 \mathrm{GeV}$, the upper bounds on the spin-independent cross-section are within reach of current or future sensitivities of direct detection experiments. For a more detailed discussion see [40]. Depending on the details of the DM model one can also perform a similar analysis to correlate observations in WEP tests and astrophysics and cosmology with collider signals. One such example for a real scalar triplet as part of multicomponent DM was studied in [40].

As long as the DM is not sterile, the presence of a WEP violating scalar force in the dark sector will be communicated to the ordinary matter sector via virtual DM effects. Depending on the DM model, this effect can be exploited to constrain dark forces weaker than gravity through correlated observations in astrophysics, cosmology, laboratory WEP tests, DM-direct-detection experiments, and collider signals.

\section{ACKNOWLEDGMENTS}

I thank Sean M. Carroll, Michael J. Ramsey-Musolf, and Christopher W. Stubbs for their collaboration that led to the work presented here. This work was supported in part by the U.S. Department of Energy contract DE-FG0208ER41531.

\section{REFERENCES}

1. A. Bosma, Astron. J. 86, 1825 (1981).

2. V. C. Rubin, D. Burstein, J. Ford, W. K., and N. Thonnard, Astrophys. J. 289, 81 (1985).

3. W. Hu, and N. Sugiyama, Astrophys. J. 444, 489-506 (1995), astro-ph/9407093

4. W. Hu, and N. Sugiyama, Phys. Rev. D51, 2599-2630 (1995), astro-ph/9411008

5. G. Jungman, M. Kamionkowski, A. Kosowsky, and D. N. Spergel, Phys. Rev. D54, 1332-1344 (1996), astro-ph/ 9512139.

6. M. Zaldarriaga, D. N. Spergel, and U. Seljak, Astrophys. J. 488, 1-13 (1997), astro-ph/9702157 
7. D. J. Eisenstein, and W. Hu, Astrophys. J. 496, 605 (1998), a stro-ph/9709112

8. D. J. Eisenstein, et al., Astrophys. J. 633, 560-574 (2005), astro-ph/0501171

9. D. Clowe, et al., Astrophys. J. 648, L109-L113 (2006), a stro-ph/ 0608407.

10. P. Zhang, M. Liguori, R. Bean, and S. Dodelson, Phys. Rev. Lett. 99, 141302 (2007), 0704.1932

11. J. Angle, et al., Phys. Rev. Lett. 100, 021303 (2008), 0706.0039

12. Z. Ahmed, et al. (2008), 0802.3530

13. O. Adriani, et al. (2008), 0810.4995

14. S. W. Barwick, et al., Astrophys. J. 482, L191-L194 (1997), astro-ph/9703192

15. J. J. Beatty, et al., Phys. Rev. Lett. 93, 241102 (2004), astro-ph/ 0412230

16. M. Aguilar, et al., Phys. Lett. B646, 145-154 (2007), astro-ph/0703154

17. J. Einasto, et al., Astron. Astrophys. 462, 397 (2007), astro-ph/ 0604539 .

18. G. R. Farrar, and P. J. E. Peebles, Astrophys. J. 604, 1-11 (2004), a stro-ph/ 0307316

19. A. Nusser, S. S. Gubser, and P. J. E. Peebles, Phys. Rev. D71, 083505 (2005), a stro-ph/ 0412586

20. M. Baldi, V. Pettorino, G. Robbers, and V. Springel (2008), 0812.3901

21. M. Kesden, and M. Kamionkowski, Phys. Rev. D74, 083007 (2006), a stro-ph/ 0608095

22. J. Bovy, and G. R. Farrar (2008), 0807 .3060.

23. M. Kesden, and M. Kamionkowski, Phys. Rev. Lett. 97, 131303 (2006), astro-ph/ 0606566

24. M.-Y. Chou, et al. (2006), astro-ph/0605101

25. R. Bean, E. E. Flanagan, I. Laszlo, and M. Trodden, Phys. Rev. D78, 123514 (2008), 0808.1105

26. T. Damour, G. W. Gibbons, and C. Gundlach, Phys. Rev. Lett. 64, 123-126 (1990).

27. J. A. Frieman, and B.-A. Gradwohl, Phys. Rev. Lett. 67, 2926-2929 (1991).

28. B.-A. Gradwohl, and J. A. Frieman, Astrophys. J. 398, 407-424 (1992).

29. G. W. Anderson, and S. M. Carroll (1997), astro-ph/9711288

30. S. M. Carroll, Phys. Rev. Lett. 81, 3067-3070 (1998), ast ro-ph/9806099

31. L. Amendola, and D. Tocchini-Valentini, Phys. Rev. D66, 043528 (2002), astro-ph/ 0111535

32. S. S. Gubser, and P. J. E. Peebles, Phys. Rev. D70, 123511 (2004), hep-th/ 0407097

33. S. S. Gubser, and P. J. E. Peebles, Phys. Rev. D70, 123510 (2004), hep-th/ 0402225

34. O. Bertolami, and J. Paramos, Phys. Rev. D71, 023521 (2005), a stro-ph/ 0408216

35. R. Bean, E. E. Flanagan, and M. Trodden, Phys. Rev. D78, 023009 (2008), 0709.1128

36. J. M. Alimi, and A. Fuzfa, JCAP 0809, 014 (2008), 0804.4100 .

37. A. Fuzfa, and J. M. Alimi, Phys. Rev. D75, 123007 (2007), a stro-ph/ 0702478

38. A. Coc, K. A. Olive, J.-P. Uzan, and E. Vangioni, Phys. Rev. D79, 103512 (2009), 0811.1845

39. S. M. Carroll, S. Mantry, M. J. Ramsey-Musolf, and C. W. Stubbs (2008), 0807.4363

40. S. M. Carroll, S. Mantry, and M. J. Ramsey-Musolf (2009), 0902.4461

41. S. Schlamminger, K. Y. Choi, T. A. Wagner, J. H. Gundlach, and E. G. Adelberger, Phys. Rev. Lett. 100, 041101 (2008), 0712.0607

42. J. G. Williams, S. G. Turyshev, and J. Murphy, Thomas W., Int. J. Mod. Phys. D13, 567-582 (2004), gr-qc/0311021.

43. e. . Lammerzahl, C., e. . Everitt, C. W. F., and e. . Hehl, F. W. (????), prepared for 220th WE-Heraeus Seminar on Gyros, Clocks, and Interferometers: Testing General Relativity in Space, Bad Honnef, Germany, 22-27 Aug 1999.

44. N. A. Lockerbie, Nucl. Phys. Proc. Suppl. 61B, 3-5 (1998).

45. M. Cirelli, N. Fornengo, and A. Strumia, Nucl. Phys. B753, 178-194 (2006), hep-ph/0512090

46. V. Barger, P. Langacker, M. McCaskey, M. J. Ramsey-Musolf, and G. Shaughnessy, Phys. Rev. D77, 035005 (2008), 0706.4311

47. X.-G. He, T. Li, X.-Q. Li, J. Tandean, and H.-C. Tsai (2008), 0811.0658. 